\title{
Los estudios de público como herramientas para analizar la relación entre sociedad y patrimonio: el caso del Museo [arqueológico] del Área Fundacional de Mendoza, Argentina
}

\author{
Florencia Puebla Antequera
}

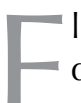

I conocimiento del patrimonio cultural, y propiamente el histórico y arqueológico, constituye una de las principales herramientas del proceso mediante el que las sociedades no sólo se identifican con su realidad y pasado sino también se legitiman a través de ese reconocimiento (cfr. Vargas Arenas y Sanoja 1993), ya que ayuda a mantener viva la memoria material e inmaterial de los pueblos y enriquece el contexto sociocultural en el que se encuentran. De ahí la necesidad de que las personas entren en contacto y se vinculen con su patrimonio, así como que se reconozcan en él (Lorenzo 1976).

Actualmente, el espacio por excelencia de exhibición del patrimonio cultural es, sin duda, el museo (Londoño 2000:6). Por ende, éste y la arqueología están en íntima conexión, y se necesitan en su mutua labor de difundir y preservar el patrimonio arqueológico (cfr. Bruno Oliveira 1999). Para que un museo que exhibe este tipo de patrimonio desempeñe eficazmente esta tarea, tiene que generar una visión crítica del pasado (Ruiz Zapatero 2002:22) que, sin distinción de realidades socioeconómicas e intelectuales, aporte a la formación de la identidad y a la transformación social de los pueblos (cfr. Barretto 2009; Batallán 1993; Gamboa Fuentes 2000): tal es la función y compromiso social de los museos arqueológicos (cfr. Delfino y Rodríguez 1997; Vázquez Olvera 2008), que, de no concretarse, se convertirán en espacios aislados del contacto e interés de las poblaciones (cfr. Dujovne 1995; Dujovne et al. 2001). En el nexo entre el pasado arqueológico y la sociedad actual, a los museos también se los considera una importante herramienta educativo-reflexiva, ya que establecen, tanto en el tiempo como en el espacio, una constante interacción entre los procesos, productos y prácticas de sociedades pasadas y presentes (cfr. Pereyra 2006).

Los museos de arqueología transmiten un discurso del pasado que, en forma diferida, es, asimismo, un discurso sobre el presente (Delfino y Rodríguez 1992:4), en el cual la forma de exponer el patrimonio material resulta clave para su interpretación y transformación (Tamanini 1999:2). Por lo anteriormente 
expuesto, vemos la importancia y necesidad de conocer y analizar la conexión que las personas crean con el patrimonio exhibido en recintos de esta naturaleza. Con base en la idea que hemos seguido hasta aquí, se realizó un estudio de público en el Museo del Área Fundacional de la Ciudad de Mendoza (MAF), ubicado al centro-oeste de la República Argentina. Creemos que meditar sobre ello desde una perspectiva crítica nos llevará a reconsiderar nuestra labor museológica y a plantear qué museos queremos tanto hoy como en el futuro.

\section{Concepción y práctica del museo desde la museología crítica}

Cuando hablamos de museos arqueológicos lo hacemos desde la mirada de la museología crítica (Lorente Lorente 2006; Navarro 2006a, 2008; Shelton 2011), la cual, en contraposición a la museología tradicional, se fundamenta y posiciona para redefinirse y concebirse a partir de un nuevo papel político-social (cfr. Lorente Lorente 2006, 2007) que supere la función comunicativa y difusionista aportada por la nueva museología (Montero 2007:1). Trabajamos con esta corriente teórica por razón de que sus ideas, aportes y alcances trascienden la mera institución museística, centrándose en la función social que ocupa hoy por hoy. Entendemos los museos como una herramienta de transformación y construcción social (cfr. Jaramillo Ferrer 2007; Navarro 2008) que, mediante la creación de una conciencia colectiva del pasado, incita el desarrollo y bienestar de los pueblos (cfr. Nascimento Junior 2008) con el fin de generar su participación social y política (cfr. Navarro 2006a). Por consiguiente, la museología crítica supone un cambio innovador dentro de los principios que fundamentan y guían a las instituciones museológicas.

Dicha corriente enfatiza, de un lado, un nuevo museo que conozca y reflexione sobre la o las construcciones que las sociedades realizan sobre su pasado (Montero 2007:3) y, del otro, cómo éstas inciden y aportan, desde la teoría del constructivismo, en el fomento al pensamiento crítico (cfr. Navarro y Tsagaraki 2009-2010). En estas construcciones las exhibiciones y el mensaje museológico juegan un papel central, porque unas y otro, con su carga política e ideológica (Pardo 2000), son los medios por los que el patrimonio llega a las personas y es interpretado por ellas. A diferencia de la nueva museología, que discurre que los museos son lugares culturales que el público visita en su tiempo libre con un fin lúdico-educativo (Declaración de Quebec 1984), para la museología crítica estas instituciones son espacios donde se hacen explícitas las controversias, subjetividades y redefiniciones culturales coexistentes en la actualidad (cfr. Padró 2003). En este proceso, enmarcado en la práctica de la enseñanza no formal (cfr. Flores Crespo 2006), las colecciones (arqueológicas en este caso) entablan un diálogo educativo y dialéctico con las sociedades por medio del que se conforma una comunidad de aprendizaje (Montero 2007:11). La finalidad, entonces, es promover valores que consoliden una ciudadanía más democrática y participativa, sin ningún tipo de distinciones (cfr. Arias Serrano 1990); es decir, que concibamos los museos como instituciones al servicio de la comunidad, donde lo que prima es un interés por conocer a sus visitantes, y las necesidades y demandas de éstos (cfr. García Pastor 2003), objetivos que la nueva museología no ha previsto ni concretado hasta la fecha (cfr. Díaz Balerdi 2002).

Es nuestra percepción que los museos deben manifestar con claridad los dilemas de la sociedad contemporánea a través de los ojos de la historia y de la memoria crítica (Navarro 2006b:4), con el fin de activar la participación política de los pueblos, y que crear - tanto dentro como fuera de éstos- espacios en los que las personas puedan generar ideas, emociones y vivencias afectivas, sociabilizadoras y reflexivas que las vinculen significativamente con su patrimonio cultural es conveniente para alcanzar dicho propósito. Desde esta perspectiva, el museo se construye con la finalidad de ser un lugar interrelacional, donde el público deja de ser un mero agente pasivo, que solamente contempla y consume, para convertirse en un protagonista activo que participa, crítica y reflexivamente (cfr. Flores Crespo 2006; Lorente Lorente 2006, Valle Flórez 2005). Estos visitantes construyen y establecen prácticas, hábitos y significados (cognitivos y culturales) con los que interpretar el patrimonio cultural y el mensaje que brinda el museo (cfr. Cousillas 1997:2), por lo que visitarlo se estima como una experiencia de disfrute y aprendizaje (Navarro 2006a:3; Navarro y Tsagaraki 2009-2010:5) mediante la cual el conocimiento y la difusión del patrimonio generan y alientan en la sociedad un ejercicio de apropiación y reelaboración de su cultura $y$, con ello, el surgimiento de una conciencia patrimonial (cfr. Dujovne 1995).

Creemos, no obstante, que para que el público participe dentro del museo es imprescindible que éste tenga una visión amplia en lo que se refiere al patrimonio, así como una cabal comprensión de los intereses y necesidades de la comunidad (cfr. Dersdepanian 2000), amplitud que ha de expresarse en políticas concretas que hagan más accesible el conocimiento y disfrute del patrimonio para aquellas personas que estaban excluidas de estas instituciones, además de tomar en cuenta diversas miradas e ideas que las exposiciones museológicas tradicionalmente marginaban. Es decir, lo que se propone es convertir el museo en espacios democráticos, representativos y polémicos (cfr. Sarno et al. 2006) donde se vean incluidos diversos puntos de vista y concepciones (Castañeda Alañón 2002:3). Afirmar que los visitantes conciben dicho patrimonio y se relacionan con él de muy diferentes maneras es comprender que dentro de los museos se entablan diálogos heterogéneos entre éste, las exposiciones y las personas. Con ello dejamos de verlos y pensarlos como lugares de contemplación y observación pasiva, 
para saludarlos como lugares complejos y dinámicos en los cuales interactúan el público, las colecciones y la museología; dicho de otra manera, los entendemos como espacios donde la gente vive experiencias y sensaciones, y crean recuerdos que establecen y afianzan lazos de pertenencia con su patrimonio e historia. Cuando acudimos a un museo, se produce una actividad socializante en la que se construyen y redefinen nuevas formas de percibir la realidad y el pasado (cfr. Cousillas 1997), por ello creemos que el visitarlo es, esencialmente, un acto social (Lleras Figueroa 2008:28).

A partir de estas ideas, consideramos importante llevar a cabo la propuesta planteada por la museología crítica en América Latina, porque necesitamos afianzar en esta sociedad una práctica democrática, ciudadana e inclusiva de la diversidad sociocultural de la que formamos parte. Es por esto que nos parece imprescindible generar museos que inciten a una actitud participativa y protagónica de los visitantes, donde se promuevan, desde el constructivismo, la subjetividad y el pensamiento crítico, y sociedades más igualitarias, participativas y democráticas (cfr. Lorente Lorente 2006; Navarro y Tsagaraki 20092010). Ante este desafío, tenemos que replantear nuestra labor profesional y reflexionar sobre el papel que otorgamos a los visitantes cuando recorren los museos, tarea para la que una herramienta válida e indispensable son los estudios de público. Definimos éstos como un proceso de relación, diálogo y análisis entre todo el conjunto de profesionales que trabajan en un museo (museólogos, curadores, arqueólogos, historiadores, etc.) y las personas que visitan el patrimonio expuesto (Puebla 2013:54). Así, partiendo de estas concepciones, realizamos un estudio de público en el MAF.

\section{El Museo del Área Fundacional (MAF)}

El Museo [arqueológico] del Área Fundacional (MAF), inaugurado en 1993, se ubica en la cuarta sección de Mendoza, dentro del casco histórico donde, sobre las ruinas de lo que fue su antiguo cabildo, se erigió la ciudad: un fuerte terremoto destruyó todo este sector urbano en 1861, lo que obligó a que la nueva urbe se trasladara hacia el suroeste, donde tiene su actual emplazamiento. Tras el sismo, el espacio perteneciente al cabildo fue reutilizado por un matadero de animales para consumo doméstico y, posteriormente, desde 1940-1950 hasta la apertura del museo, por un mercado de frutas y verduras (cfr. Bárcena y Schávelzon 1991; Chiavazza 2011).

Excavado por primera vez en 1989 (cfr. Bárcena y Schávelzon 1991), el sitio arqueológico reveló importantes hallazgos, que condujeron a crear el museo de sitio (cfr. Tamiozzo 1997) (Figura 1).

Hoy por hoy, este museo abre 323 días al año y, de martes a sábados, durante 12 horas corridas: de las 8 de la mañana a las 8 de la noche; los domingos atiende de 12 a 8 y los días feriados, de 11 a 7 . El costo de ingreso varía, si también se quiere ingresar en la cámara subterránea, y según el público: el general, los estudiantes y los niños menores de ocho años pagan tarifas diferenciadas, mientras que los jubilados tienen entrada libre; los miércoles el acceso es gratuito indistintamente del tipo de visitante.

La muestra que esta institución presentó en el momento en que se realizó el presente estudio estaba conformada por tres salas de exposiciones permanentes y una de temporales.

El guión museológico del MAF — hasta ese momento en idioma español- estaba basado en la historia de la ciudad de Mendoza, dividida en periodos históricos/ arqueológicos característicos en la región: indígena, colonial y nueva ciudad (posterremoto). La exposición que actualmente se exhibe, renovada completamente en $2008^{1}$ tras la asunción del actual director del MAF, el doctor en arqueología Horacio Chiavazza, contiene dos grandes componentes: por un lado las colecciones, en su mayoría arqueológicas, históricas y etnográficas, y por el otro la exhibición de excavaciones arqueológicas, que incluye la vista de los pisos originales del cabildo de la ciudad.

Por la importancia del museo, así como del patrimonio local y regional que alberga, decidimos realizar ahí el primer estudio sistemático de público.

\section{Estudio de público en el MAF}

Llevado a cabo en el año 2011, el estudio de público en el MAF buscó conocer y analizar la forma en que los visitantes se relacionaban con las colecciones en exhibición. Para ello se aplicaron técnicas metodológicas utilizadas en otros trabajos a escala mundial, con adecuaciones propias a las problemáticas del caso en particular.

\section{Clasificación de variables utilizadas en el público}

Para estudiar y analizar a los visitantes del MAF, empleamos las siguientes variables:

- Edad: Basada en las etapas aportadas por la teoría constructivista (cfr. Piaget 2005), las cuales definimos en subgrupos categóricos propios, de acuerdo con el desarrollo motivacional y cognitivo de las personas:

\begin{tabular}{|c|c|}
\hline $\begin{array}{r}\text { Etapa de } \\
\text { operaciones } \\
\text { concretas }\end{array}$ & $\begin{array}{l}\text { 7-11: denominados niños. } \\
\text { 12-17: denominados adolescentes. } \\
\text { 18-28: denominados jóvenes. }\end{array}$ \\
\hline $\begin{array}{r}\text { Etapas de } \\
\text { operaciones } \\
\text { formales }\end{array}$ & $\begin{array}{l}\text { 29-40: denominados adultos de tipo } 1 \\
\text { 41-64: denominados adultos de tipo } 2 \\
65 \text { en adelante: denominados jubilados }\end{array}$ \\
\hline
\end{tabular}

${ }^{1}$ Para tener conocimiento sobre la exposición anterior a 2008, véase Chiavazza (2011). 


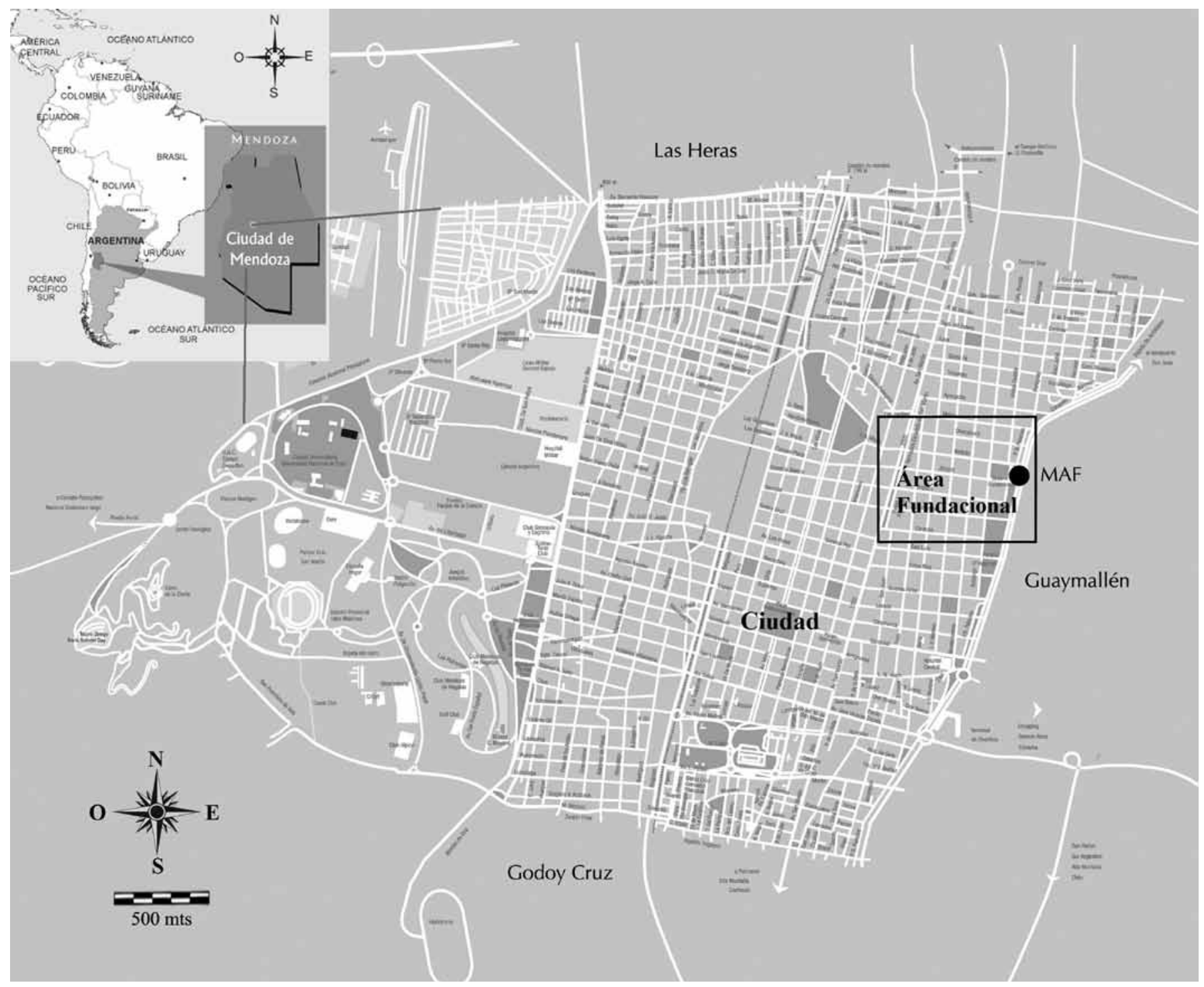

FIGURA 1. Ubicación del MAF en la ciudad de Mendoza, Argentina (Cortesía: Florencia Puebla Antequera, 2011).

- Sexo: Femenino Masculino

- Lugar de origen: Discriminado por grupos de procedencia que correspondían a los diferentes continentes (África, América, Asia, Europa y Oceanía); se incorporaron categorías locales: de Argentina y de Mendoza.

Respecto de la caracterización de las visitas, se las discriminó por tipos, correspondientes a las actividades, particularidades, causas e intereses que las conforman. Éstas son:

- Educativa de origen Mendoza

- Educativa de otras provincias de Argentina

- Turística de origen Mendoza

- Turística de otras provincias de Argentina
- Turística de otra nacionalidad

- Visita especial (ingresan en el museo por una causa en particular o para presenciar un tipo de actividad diferente de los demás tipos de visita)

Periodo de toma y recopilación de datos

Para cada una de las técnicas se seleccionó un periodo específico de desarrollo (Figura 2):

Cálculo y resultados del universo muestral

Para estimar el tamaño muestral, se tomaron de referencia los totales mensuales del año 2010. Para ello se seleccionaron los meses que presentaban los niveles más altos de visitas: junio (1 904 visitantes), julio (1 664), agosto (2654), septiembre (3258), octubre (4115) y noviembre (3949). Con estas cifras calculamos la muestra 


\begin{tabular}{|c|c|}
\hline TIPO DE TÉCNICA & $\begin{array}{c}\text { MESES A LLEVARLAS A } \\
\text { CABO EN EL AÑO 2011 }\end{array}$ \\
\hline Observación directa & Junio-Julio \\
\hline $\begin{array}{c}\text { Mapeos de significado } \\
\text { personal }\end{array}$ & Agosto \\
\hline $\begin{array}{c}\text { Encuestas actitudinales } \\
\text { Cuestionarios } \\
\text { auto-administrados }\end{array}$ & Septiembre-Octubre \\
\hline $\begin{array}{c}\text { Conversaciones } \\
\text { provocadas }\end{array}$ & Noviembre \\
\hline $\begin{array}{c}\text { Relevamientos del libro } \\
\text { de visitas }\end{array}$ & Diciembre \\
\hline
\end{tabular}

FIGURA 2. Desarrollo de las técnicas metodológicas en el año 2011 (Cortesía: Florencia Puebla Antequera, 2011).

representativa con la fórmula de población finita (cfr. Badii et al. 2011), utilizada para un muestreo de características probabilístico (Casal y Mateu 2003) (Figura 3):

$$
n=\frac{k^{\wedge 2 *} p^{*} q^{*} N}{\left(e^{\wedge 2 *}(N-1)+K^{\wedge 2 *} p^{*} q\right.}
$$

FIGURA 3. Fórmula de población finita (Cortesía: Florencia Puebla Antequera, 2011).

\section{Desarrollo de la fórmula}

n: es el resultado que nos da el tamaño de la muestra ficticia que tomaremos en cada mes.

k: es la constante que depende del nivel de confianza asignado a la muestra, el cual indica la probabilidad de que los resultados de nuestra investigación sean ciertos. En este caso, se tomó 95 por ciento.

p: es la proporción de individuos que en la población poseen la característica de estudio. Este dato es generalmente desconocido y suele suponerse que $p=q=0.5$, que es la opción estadísticamente más segura.

q: es la proporción de individuos que no poseen esa característica; se calcula 1-p. e: es el error muestral deseado, es decir, la diferencia que puede haber entre el resultado que obtenemos de la muestra y el total de la población que, en este caso, es el total de los visitantes del mes. Se tomó un error de 2 por ciento.

$\mathrm{N}$ : es el tamaño de la población o universo. Éste, en nuestro cálculo, es el número total de visitantes por mes elegido del año 2010; estos totales varían mensualmente.

Error muestral: Nivel de confianza de la muestra: 95\% Error máximo: $\pm 2 \%$

La decisión de emplear esta fórmula respondió a que representa un margen de error mínimo, por lo que es la más socorrida en investigaciones de índole científica. Al aplicarla, obtuvimos los siguientes cálculos, que nos permitieron deducir la muestra representativa 2011 (Figura 4):

Universo de la muestra: En total se analizaron 2027 personas que visitaron el MAF en 2011. Es importante aclarar que se puso especial énfasis en que las personas analizadas presentaran diversidad de orígenes, edad y sexo, con la finalidad de que la muestra fuese lo más incluyente y representativa posible.

Técnicas metodológicas utilizadas

Observaciones directas: en busca de una mayor fiabilidad en los comportamientos de las personas, se cuidó particularmente que los visitantes no advirtieran que se les observaba en el espacio expositivo. Se eligieron puntos de muestreo (lugar donde el observador se ubica para registrar los comportamientos) que ofrecieran amplia visibilidad, para abarcar todos los rincones de cada sala.

Mapeos de significado personal (PMM, por sus siglas en inglés): dicha técnica consistió en que el visitante, tanto antes de entrar (primer momento de Ilenado de PMM) como al salir del museo (segundo momento) contestara oraciones que tenían que ver con éste, su temática y colecciones. Posterior a ello se realizaba una pequeña entrevista personal, para que el visitante explicara lo escrito en el PMM (tercer momento). El objeto de este ejercicio era poder observar y registrar cambios actitudinales referidos a lo que se preguntaba una vez concluida la experiencia museística dentro del MAF. Dichos cambios se consignaban en lo que anotaban en el segundo momento, es decir, si cambiaban su parecer con lo completado en el primer momento o si mantenían su idea (Figura 5).

Encuestas actitudinales: realizadas una vez concluido el recorrido por el museo, estaban orientadas a analizar si la visita y la experiencia museística que éste conlleva producía cambios en los visitantes respecto de las ideas, 


\begin{tabular}{|l|c|c|c|c|c|c|}
\hline \multicolumn{1}{|c|}{ TIPO DE TÉCNICA } & JUNIO & JULIO & AGOSTO & SEPTIEMBRE & OCTUBRE & NOVIEMBRE \\
\hline Educativas de Mendoza & 129 & 46 & 80 & 111 & 128 & 190 \\
\hline Educativas de Argentina & 47 & 31 & 80 & 103 & 53 & 29 \\
\hline Turísticas de Argerntina & 21 & 142 & 60 & 22 & 25 & 21 \\
\hline Turísticas de Mendoza & 24 & 45 & 50 & 44 & 70 & 54 \\
\hline Turísticas de americanos & 9 & 21 & 50 & 21 & 16 & 18 \\
\hline Turístivas de europeos & 4 & 17 & - & 4 & 6 & 7 \\
\hline Visitas especiales & 73 & - & - & 31 & 37 & 31 \\
\hline Total de muestra a realizar en el mes & $\mathbf{3 0 7}$ & $\mathbf{3 0 2}$ & $\mathbf{3 0 0}$ & $\mathbf{3 3 6}$ & $\mathbf{3 3 5}$ & $\mathbf{3 5 0}$ \\
\hline
\end{tabular}

FIGURA 4. Muestra representativa del año 2011, dividida según los tipos de visitas (Cortesía: Florencia Puebla Antequera, 2011).

sensaciones y opiniones que les generaba la muestra (Figura 6).

Cuestionarios autoadministrados: se completaron una vez finalizado el recorrido. En un primer momento se tomaron variables informativas sociodemográficas, más otras que tenían que ver con el museo en general: sus servicios, la exposición museográfica y las excavaciones arqueológicas. También se tomaron datos que hacían referencia al número y frecuencia de visitas previas, la compañía durante el recorrido, cuáles fueron los objetos y vitrinas que más Ilamaron la atención (focos de atención), la comprensión de contenidos generales y la asimilación de ideas básicas del guión museológico (Figura 7).

Conversaciones provocadas: esta técnica constó de un ejercicio de entrevista aplicado a un pequeño grupo de visitantes (de no más de cuatro personas) una vez finalizado el recorrido. En un primer momento se registraban datos sociodemográficos;

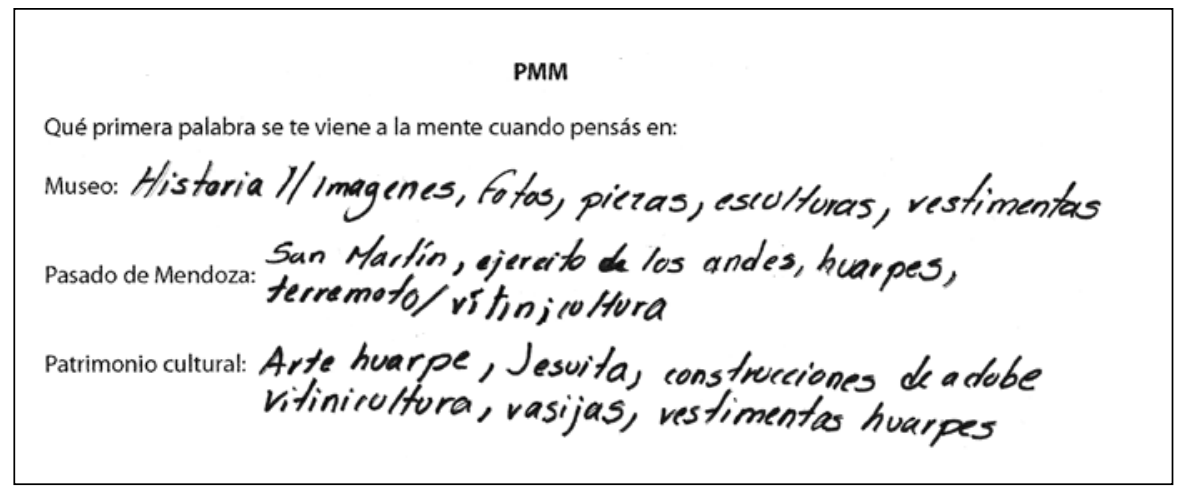

FIGURA 5. Mapeo de significado personal (PMM) Ilenado por un visitante del MAF (Florencia Puebla Antequera, 2011; cortesía: MAF).

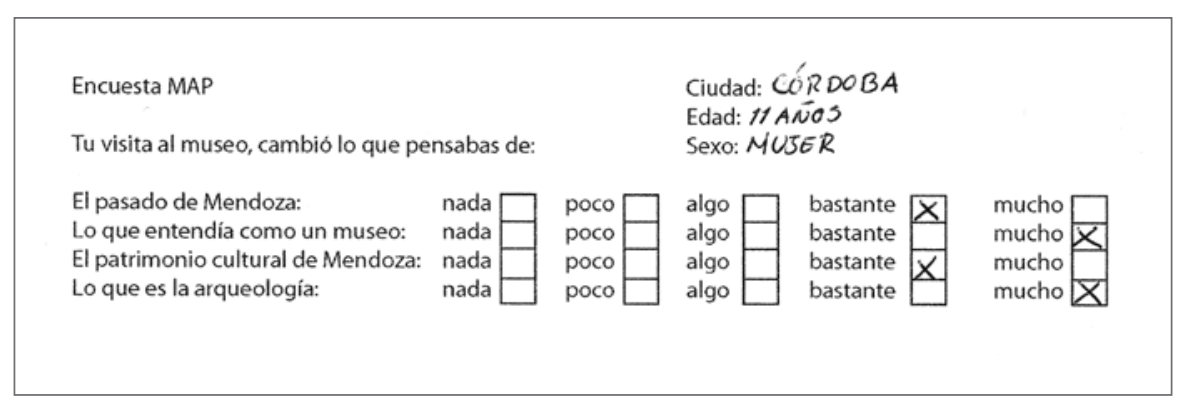

FIGURA 6. Encuesta de actitud completada por un visitante del MAF (Florencia Puebla Antequera, 2011; cortesía: MAF). 


\section{ENCUESTA DE PÚBLICO DEL MUSEO DEL ÁREA FUNDACIONAL}

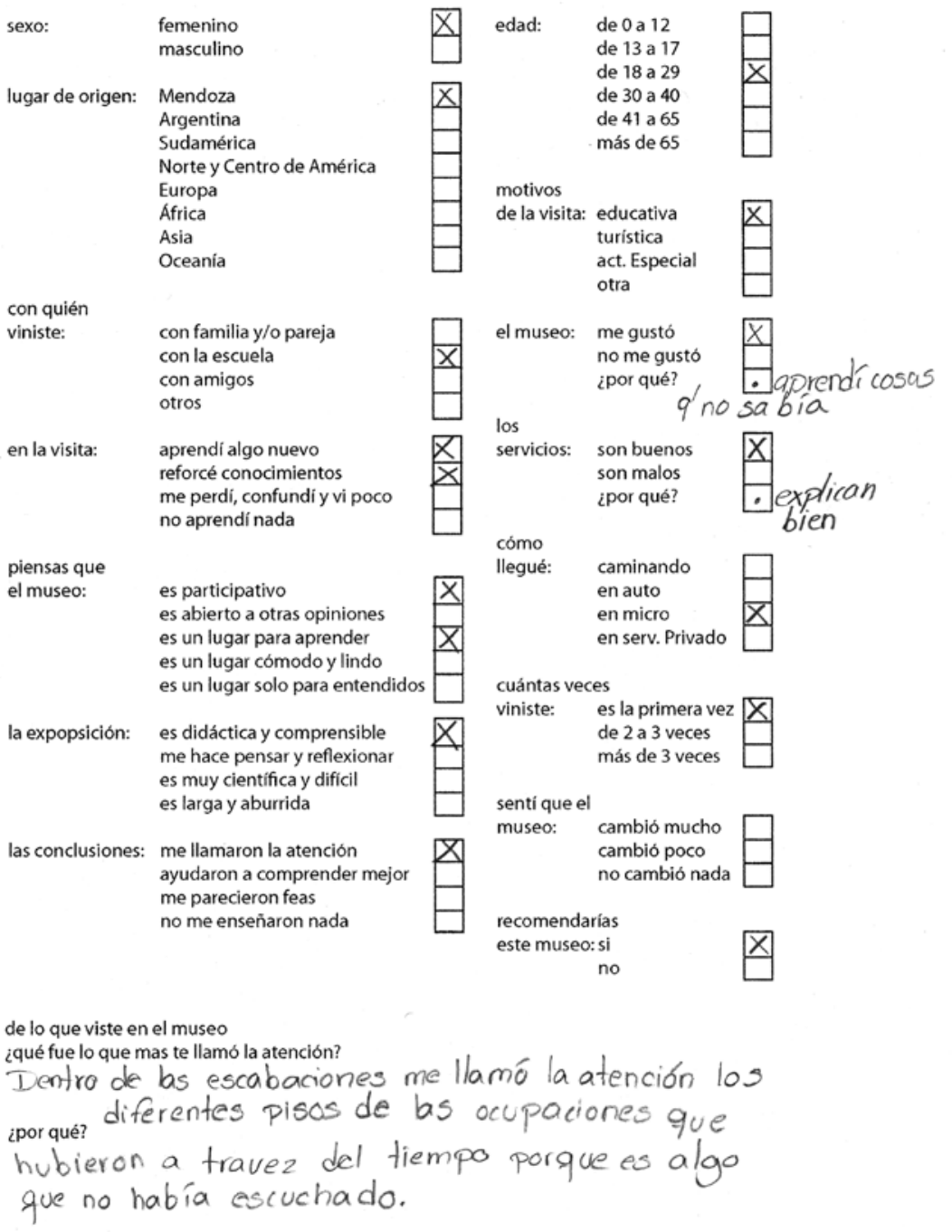

FIGURA 7. Cuestionario autoadministrado (Florencia Puebla Antequera, 2011; cortesía: MAF).

después, se realizaban preguntas sobre qué les habían parecido el museo, la exposición y las excavaciones. Nos focalizamos, entonces, en saber cómo los visitantes habían percibido el patrimonio expuesto en el MAF e interactuado con él, para lo que se realizó un ejercicio consistente en que el entrevistado tratara de ubicar en el tiempo y en las salas de exposición diversos objetos arqueológicos, su utilización y lo que les había provocado. La finalidad de dichas preguntas era afianzar conocimientos y poder registrar si los visitantes habían adquirido datos nuevos. Por último, se indagó sobre la experiencia de cada uno de ellos en particular; se preguntaba, por ejemplo, cuáles habían sido las sensaciones y emociones generadas por los materiales expuestos cuando ingresaron, recorrieron y egresaron del museo, así como también qué opinión les provocaban éstos.
Relevamiento del libro de visitas: se documentó todo lo que los visitantes del MAF apuntaron desde el 1 de enero hasta el 31 de diciembre de 2011, con el fin de conocer las opiniones que dejaban por escrito.

Finalizado el trabajo de campo, la información obtenida de cada técnica metodológica se procesó, y se procedió a analizar cómo era la visita que las personas efectuaban en el MAF para, a partir de ahí, reflexionar sobre nuestra labor museológica.

\section{Conclusiones aportadas por el estudio de público ${ }^{2}$}

Ya expuestos los principios teóricos que guían nuestro trabajo y los métodos empleados para la obtención de datos, discutiremos de forma breve y acotada, ${ }^{3}$ algunos de los resultados del estudio de público.

El trabajo realizado nos demostró, primeramente, que los visitantes del MAF poseen gran diversidad en su perfil sociodemográfico, lo cual indica que es una institución a la que concurren públicos de gran heterogeneidad en cuanto a edad, origen y grado de educación. Asimismo, se evidenció que tanto los niños como los adolescentes que acuden con fines educativos desarrollan un aprendizaje de la historia de la ciudad en un contexto lúdico y reflexivo, que genera una mejor comprensión de los saberes adquiridos. Esto se comprobó en los PMM, en las conversaciones provocadas, así como en las observaciones, ya que daban cuenta de que las visitas se realizaban en un ambiente de aprendizaje a través del juego, donde este tipo de población, en grupo, participaban, se divertían, aprendían y reflexionaban sobre el pasado de Mendoza. Pese a ello, no fue únicamente este segmento el que aprendió nuevos conocimientos: todos incorporaron nueva información sobre este tema una vez que concluyeron su visita, independientemente de su perfil sociodemográfico, según lo reflejan de las encuestas actitudinales, los PMM y

\footnotetext{
${ }^{2}$ De aquí en adelante, expresiones como todos, grupos, niños y adolescentes, personas, se refieren a los visitantes que en su totalidad acuden al Museo del Área Fundacional de Mendoza (MAF).

${ }^{3}$ Para consultar todos los datos obtenidos y las conclusiones realizadas en dicho estudio de público, véase Puebla (2013).
} 
el relevamiento del libro de visitas. Este dato hace patente que tanto la exposición como su guión museológico son entendibles para todos, sin que sean condicionantes su grado de estudios, procedencia o edad. No obstante, durante su recorrido por el MAF el público, en un diálogo que se desarrolla en una relación dialéctica, aporta datos y comentarios muy útiles y valiosos tanto para el museo, en la medida en que ayudan a los profesionales de éste a mejorarlo, como para quienes asisten a él (Figura 8).

Además, las observaciones realizadas demostraron gran variedad no sólo de tipos de recorridos y circulación dentro del museo sino también de lecturas y puntos de atención por parte de los visitantes a la exposición, diversidad que se relaciona con los perfiles, edades, intereses y gustos de las distintas personas que ingresan diariamente en el MAF. Éste, según los datos obtenidos en tal aspecto, está montado y diseñado para que lo frecuenten y disfruten diversos públicos; es decir, constituye un espacio donde claramente se evidencia la diversidad sociocultural, propia de la realidad en la que vivimos.

Aunado a lo anterior, se comprobó que $87 \%$ de los asistentes analizados registró fatiga museal o atencional $^{4}$ al concluir la travesía, porcentaje que nos obliga a revaluar el diseño expositivo, así como la ubicación de espacios de descanso dentro del sitio, con la finalidad de que no se haga tedioso y largo recorrerlo.

${ }^{4}$ Véase Colino Polo y De la Peña Alonso (2005), donde se da a conocer y se analiza el concepto de fatiga museal.
Los PMM y las encuestas, tanto actitudinales como autoadministradas, también revelaron que $77 \%$ de los visitantes reflexionó sobre la historia de la ciudad y el cuidado del patrimonio cultural. Esto lo confirman muchas frases y opiniones aportadas por los públicos, que asentaron que el museo les había hecho descubrir, conocer, pensar y valorar la riqueza cultural material de la ciudad y la importancia de protegerla. Los mapeos revelaron que, al término de la visita, $96 \%$ experimenta cambios conceptuales y actitudinales sobre lo aprendido. Ejemplo de ésto fue que muchos individuos se emocionaron frente a ciertos hechos históricos, como el sometimiento de los pueblos originarios de la zona durante la época colonial o el terremoto que destruyó la ciudad: que experimenten sentimientos sobre su pasado genera empatía histórica, así como un acercamiento e identificación personal con éste, por lo que es una cualidad muy importante del museo. Adicionalmente se observó, en $42 \%$ de los visitantes analizados, que adultos mayores mendocinos y nacionales no sólo llevan a los niños a conocer o repasar el museo sino además les explican su historia de dicha ciudad. Esto es de enorme valor, en primer lugar porque se está educando a generaciones futuras en la práctica de frecuentar el sitio como una actividad familiar y cotidiana, y en segundo lugar porque los mayores, al tomar la voz y explicar sus conocimientos sobre el pasado de la región a los más pequeños, refrendan el valor del diálogo entre las personas, la exposición y el patrimonio (Figura 9).

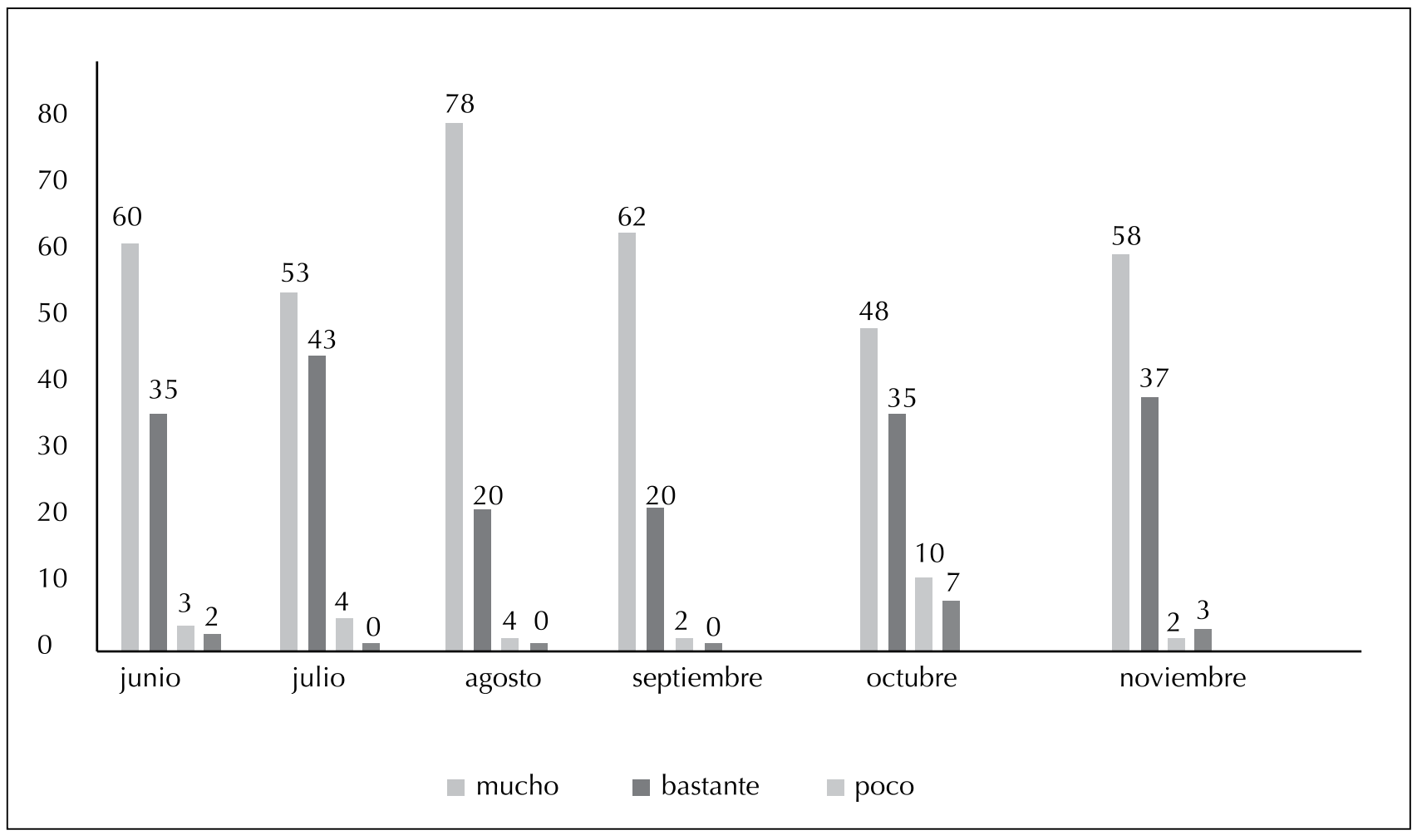

FIGURA 8. Índices de incorporación de nuevos conocimientos en los visitantes (Cortesía: Florencia Puebla Antequera, 2011). 
Las observaciones realizadas también revelaron que los visitantes, cuando recorren el museo en grupos guiados, se encuentran, comparten, conversan, discuten e interactúan con personas desconocidas, de diversos lugares y edades: en $87 \%$ de las observaciones a grupos guiados se evidenció que se producían debates sobre un particular hecho histórico o acerca de la exposición en sí. Además de ello, los cuestionarios autoadministrados y el libro de visitas dieron a saber que todos se sintieron suficientemente cómodos dentro del MAF, y que pudieron expresarse libremente durante el recorrido tanto con quienes los acompañaban como con desconocidos, lo cual demuestra que aquél es un espacio donde las personas se sienten libres de expresar su opinión, y de atender puntos de vista y formas de pensar diferentes. Creemos que estas prácticas son significativas, ya que promueven valores democráticos, importantes en la sociedad actual, como son la participación y el debate en espacios públicos; es decir, la visita a este museo no sólo enseña sobre el pasado de la ciudad de Mendoza sino también promueve y practica valores que llevan a mejorar la convivencia ciudadana presente.

Respecto de los datos recabados del libro de visitas, 928 personas decidieron escribir 830 comentarios que en su mayoría (96\%) fueron positivos, lo que nos indica que el porcentaje de inconformidad no es alto (4\%), considerando que la mayor parte de quienes se quejan

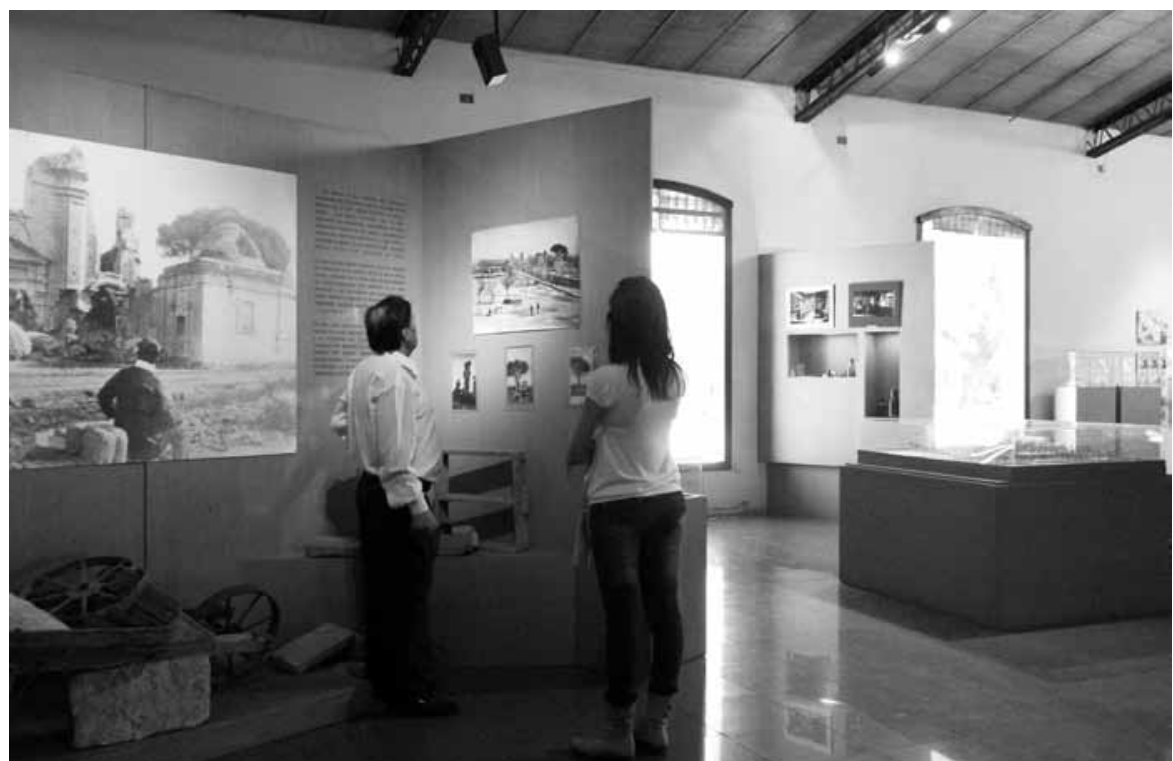

FIGURA 9. Visitantes recorriendo la muestra del MAF (Fotografía: Florencia Puebla Antequera, 2011; cortesía: MAF)

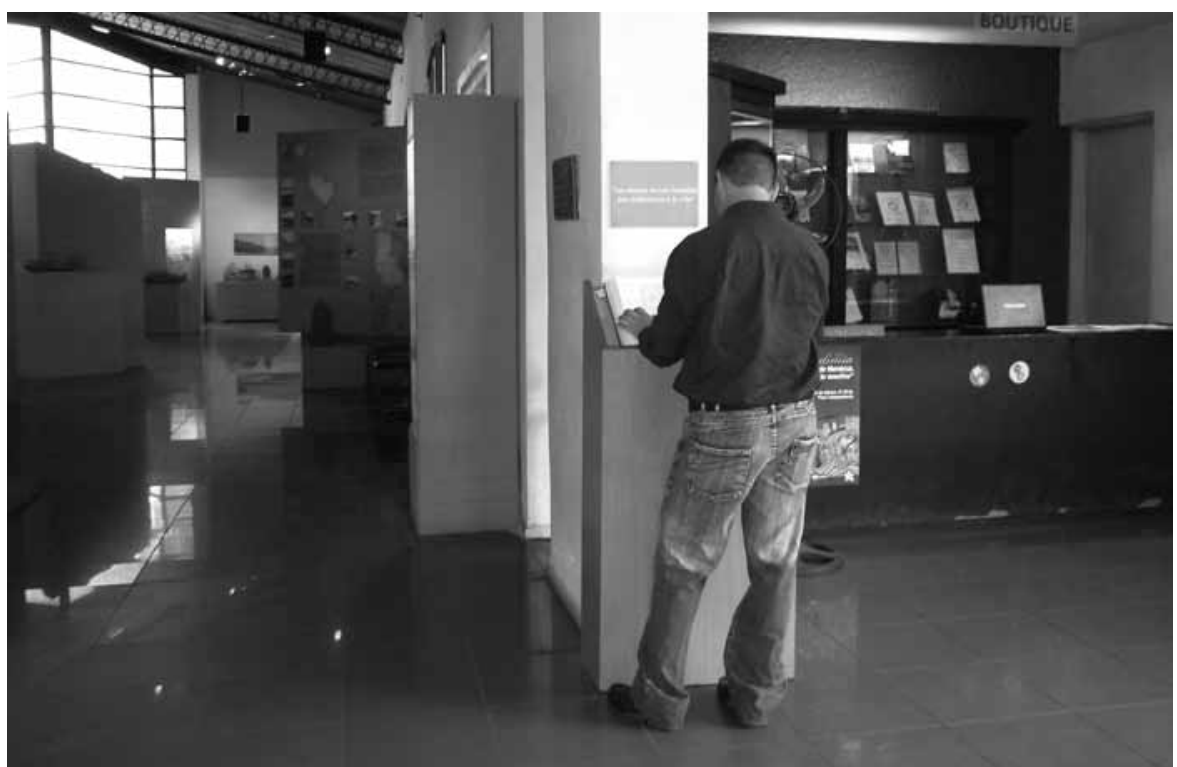

FIGURA 10. Visitante escribiendo en el libro de visitas (Fotografía: Florencia Puebla Antequera; cortesía: MAF). o manifiestan una opinión negativa del museo lo hace por escrito en dicho libro, transformándolo, así, en un elemento clave para mejorar la gestión museal (Figura 10).

Al mismo tiempo, por las encuestas actitudinales concluimos que las personas de las visitas de tipo educativo de Mendoza, y turístico del país, fueron las que mayormente modificaron sus concepciones sobre el pasado de la ciudad tras visitar el MAF, seguidas por aquellas de visitas educativas provenientes de diversos puntos de la Argentina. Entendemos que esto se debe a que los que no viven en esta provincia tienen poco o nulo conocimiento sobre su pasado, por lo que el MAF les enseña algo que no sabían y no habían aprendido en otro lugar —la

escuela, por ejemplo-, como sí sucede con los oriundos de Mendoza. En el caso de quienes acudieron al museo con la escuela, están aprendiendo sobre el pasado de su ciudad, por lo que el periplo ayuda en ese proceso de conformación de conceptos, aportando nuevas ideas y conocimientos que en la escuela no se brindan, como es la ciencia arqueológica, la toma de conciencia sobre la preservación del patrimonio y otras concepciones que se desarrollan concomitantemente, como es la identidad, la memoria, el pasado, etcétera.

Otra información valiosa aportada por el estudio de público es que fueron las personas de visitas educativas 
de Mendoza, y de visitas consideradas como actividades especiales, las que más cambiaron sus ideas respecto de la ciencia arqueológica. Es importante aclarar que todas ellas recorren las salas con el guía, cuya explicación sobre cómo trabaja dicha ciencia es determinante en estos cambios conceptuales. También se verificó que los mayores cambios en lo que concierne al patrimonio cultural mendocino provienen de visitantes de Argentina que conocen el museo por placer, es decir, como turistas. Esto es así ya que para éstos muchas veces se trata de su primer acercamiento al sitio, y los cambios dichos se producen dentro de éste. Adicionalmente, son los visitantes educativos de Mendoza los que mayormente cambian sus ideas respecto de lo que es un museo, ya que, por su edad, no tienen una noción formada de lo que esta institución comprende y representa.

Todas las conclusiones anteriormente mencionadas confirman que el MAF es un lugar de encuentro social, de aprendizaje y reflexión, además de un espacio de debate e intercambio de opiniones, donde se expresan diversas formas de pensar, y que desde el reconocimiento del pasado generan mayor tolerancia social. El que estas prácticas se realicen dentro del museo es muy importante tanto para la museología como para la arqueología, ya que hace explícita la relación existente entre el museo y el patrimonio allí exhibido con su contexto social (Figura 11).

\section{Consideraciones finales}

El trabajo realizado corroboró que los estudios de público son herramientas válidas para analizar tanto la relación que las personas tienen con los museos como la influencia científica y social que éstos ejercen en el presente. Tener conocimiento de ello nos permite, por un lado, llevar a cabo una gestión del patrimonio cultural acorde con los intereses, demandas y necesidades de la sociedad actual, y, por el otro, reflexionar acerca de la práctica museológica contemporánea. Ante este panorama, proponemos un museo social en permanente diálogo con su comunidad, con el fin de que ésta, a través de su patrimonio cultural, pueda reflexionar crítica y subjetivamente acerca de la realidad de la cual forma parte. Por ello consideramos fundamental llevar a cabo estudios de público que nos ayuden a conocer y analizar cómo es la visita que realizan las personas dentro de los museos.

El estudio evidenció, asimismo, sus puntos positivos y errores, que desconocíamos hasta el momento; reveló, además, información muy valiosa sobre cómo las personas se desenvuelven dentro del MAF. Por ello consideramos que los estudios de público, lejos de ser un fin en sí mismo, son un punto de partida para afrontar el desafío de generar museos críticos y reflexivos respecto de su presente patrimonial.

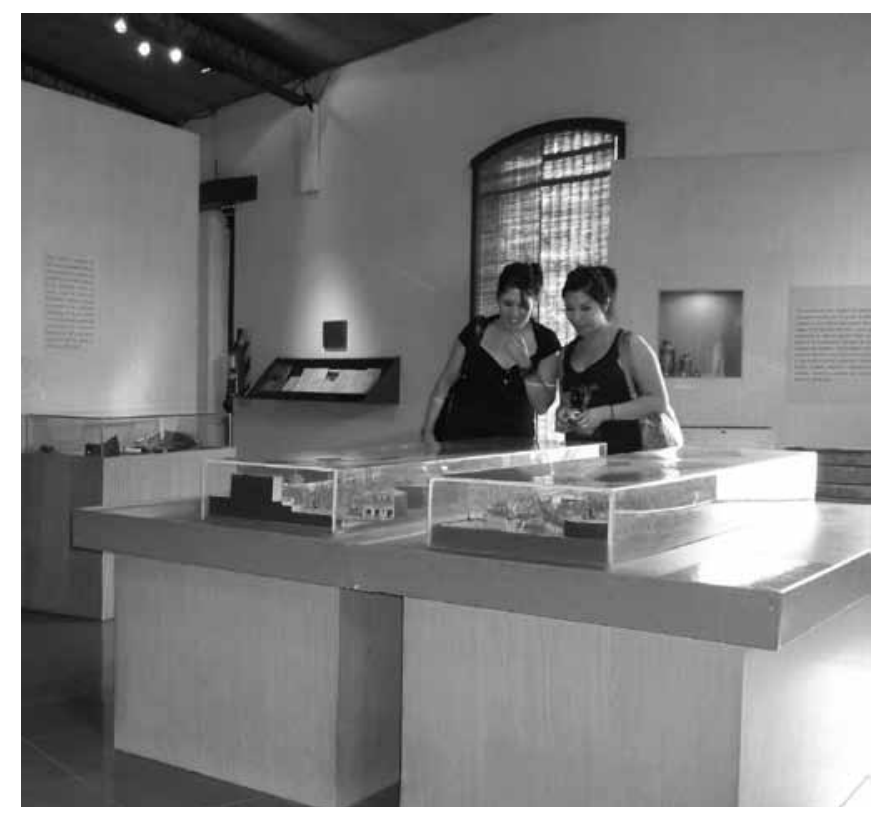

FIGURA 11. El MAF es un espacio donde se genera el encuentro entre personas (Fotografía: Florencia Puebla Antequera; cortesía: MAF).

\section{Agradecimientos}

Mi gratitud al director del MAF y tutor de mi tesis, el doctor Horacio Chiavazza, por permitirme realizar este trabajo; a los trabajadores del museo, y a todos los visitantes que amablemente participaron en el estudio de público.

\section{Referencias}

\section{Arias Serrano, Laura} 1990 "El papel del público en el museo de hoy", Boletín del Anabad, 40 (2-3): 179-188.

Badii, M. H., A. Guillen, E. Cerna y J. Valenzuela

2011 "Nociones introductorias de muestreo estadístico", Daena: International Journal of Good Conscience, 6 (1): 89105.

Bárcena, Roberto y Daniel Schávelzon 1991 El cabildo de Mendoza. Arqueología e historia para su recuperación, Mendoza, Municipalidad de Mendoza.

Barretto, Margarita 2009 "Los museos y su papel en la formación de la identidad", Prehistoria y Arqueología JVG, documento electrónico disponible en [http://prehistoriayarqueologiajvg.blogspot. com], consultado en diciembre de 2011.

Batallán, Graciela 1993 "Museos, patrimonio y educación. Reflexiones en el Museo Etnográfico Juan B. Ambrosetti", en Isabel Lamounier (ed.), Museos y sociedad, Buenos Aires, CEAL, 73-81.

Bruno Oliveira, María Cristina 1999 “A impotância dos processos museológicos para a pre- 
servaçao do patrimônio", Revista do Museu de Arqueologia e Etnologia, São Paulo, suplemento 3: 333-337.

Casal, Jordi y Enric Mateu

2003 "Tipos de muestreos", Revista de Epidemiología y Medicina Preventiva Veterinaria, 1: 3-7.

Castañeda Alañón, Lucía 2002 “¿Usuarios o visitantes?”, Revista Museo, 6: 1-13.

Chiavazza, Horacio

2011 "El Área Fundacional de Mendoza”, en M. Ramos et al. (ed.), Temas y problemas de la arqueología histórica, Buenos Aires, Universidad Nacional de Luján, II: 45-65.

Colino Polo, Fernando y Paloma de la Peña Alonso

2005 "El cansancio en los museos", Arqueoweb 7 (1), documento electrónico disponible en [http://pendientedemigracion.ucm.es/info/arqueoweb/pdf/7-1/colino.pdf], consultado en marzo del 2012.

Cousillas, Ana María

1997 Los estudios de visitantes a museos: fundamentos generales y principales tendencias, MMAJH-Secretaría de CulturaGCBA.

Declaración de Quebec 1984 Principios básicos de una nueva museología, Quebec.

Delfino, Daniel y Pablo Gustavo Rodríguez

1992 "La re-creación del pasado y la invención del patrimonio arqueológico", Publicar. Antropología y Ciencias Sociales 2, s/p. 1997 Los museos de arqueología, ausencia del presente en las representaciones del pasado, documento electrónico disponible en [www.naya.org.ar/articulos/museologia04.htm], consultado en noviembre de 2011.

Dersdepanian, Georgina

2000 "El museo comunitario, un principio para todos", Gaceta de Museos (17), 9-16.

Díaz Balerdi, Ignacio

2002 “Qué fue de la nueva museología? El caso de Quebec", Artigrama, 17: 493-516.

Dujovne, Marta

1995 Entre musas y musarañas. Una visita al museo, Buenos Aires, FCE.

Dujovne, Marta, Silvia Calvo y Verónica Staffora

2001 Ir al museo: notas para docentes, Buenos Aires, Ministerio de Educación/Unidad de Recursos Didácticos/Universidad de Buenos Aires.

Flórez Crespo, María del Mar

2006 "La museología crítica y los estudios de público en los museos de arte contemporáneo: caso del museo de arte contemporáneo Castilla y León, Musac", De arte, 5: 231-243.

Gamboa Fuentes, Sonia

2000 "Museo, museografía y museología", Biblios, Revista Electrónica de Bibliotecología, Archivología y Museología, 2 (5): 23-34.

García Pastor, Carmen 2003 "Segregación, integración e inclusión", Bordón, Revista de Orientación Pedagógica, 55 (1): 9-26.

Jaramillo Ferrer, Carolina

2007 "Los museos como herramientas de transformación social del territorio. El caso del Museo de Antioquia, Medellín,
Colombia", documento electrónico disponible en [http:// www.uv.es/museos/MATERIAL/Jaramillo.pdf], consultado en noviembre de 2011.

Lleras Figueroa, Cristina

2008 "¿Objetos demodé? Museos y patrimonio intangible", Calle 14: Revista de Investigación en el Campo del Arte, 2: 22-29.

Londoño, Eduardo

2000 "Una exhibición de arqueología en diálogo con el público", Museo del Oro, Banco de la República, documento electrónico disponible en [http://www.banrep.gov.co/museo/ esp/educa_dialogo.htm], consultado en junio de 2012.

Lorente Lorente, Jesús-Pedro

2006 "Nuevas tendencias en la teoría museológica: a vueltas con la museología crítica", Revista Museos.es, Revista de la Subdirección General de Museos Estatales, 2: 231-243, documento electrónico disponible en [http://www.mcu.es/museos/docs/MC/MES/Rev02/Rev02_Jesus-Pedro_Lorente.pdf], consultado en diciembre de 2011.

2007 "Dos revisiones sobre la historia de la museología", RdM, Revista de Museología, 38: 134-136, documento electrónico disponible en [http://dialnet.unirioja.es/servlet/ revista?codigo=3693], consultado en diciembre de 2011.

Lorenzo, José Luis (coord.)

1976 El desarrollo de la antropología en América Latina, México, INAH.

Montero, Javier

2007 "Pedagogía crítica y educación en museos. Marcos para una educación artística desde las comunidades", en Olga Fernández y Víctor del Río (eds.), Estrategias críticas para una práctica educativa en el arte contemporáneo, Valladolid, Museo Patio Herreriano/Caja España Obra Social, 118-132.

Nascimento Junior, José do

2008 "Los museos como agentes de cambio social y desarroIlo", Museos.es, Revista de la Subdirección General de Museos Estatales, 4: 16-27.

Navarro, Jorge Fernando y Olga Nazor

2001 "La museología en la encrucijada", Biblios, Revista de Bibliotecología y Ciencias de la Información, 2, documento electrónico disponible en [http://www.redalyc.org/articulo. oa?id=16107508], consultado en diciembre de 2011.

Navarro, Óscar

2006a "Museos y museología: apuntes para una museología crítica", en Hildegard K. Vieregg, Mónica Risnicoff de Gorgas, Regina Schiller (eds.), Museología e historia: un campo de conocimiento, XXIX Encuentro Anual del ICOFOM/XV Encuentro Regional del ICOFOM LAM, 5 al 15 de octubre, Córdoba y Alta Gracia, Argentina, documento electrónico disponible en [http://www.icofom-lam.org/files/museos_y_ museologia_critica_-_copia_2.pdf], consultado en julio de 2012.

2006b "Museos nacionales y representación: ética, museología e historia", en Hildegard K. Vieregg, Mónica Risnicoff de Gorgas, Regina Schiller (eds.), Museología e historia: un campo del conocimiento, XXIX Encuentro Anual del ICOFOM/ XV Encuentro Regional del ICOFOM LAM, 5 al 15 de octubre, 
Córdoba y Alta Gracia, Argentina, 385-394.

2008 "Museología y capacitación: los retos de la enseñanza museológica vistos desde la museología crítica", Seminario Permanente de Museología en América Latina, México, ENCRyM-INAH, 15-27.

Navarro, Óscar y Cristina Tsagaraki

2009-2010 "Museos en la crisis: Una visión desde la museología crítica", Museos.es, Revista de la Subdirección General de Museos Estatales (5-6): 50-57, documento electrónico disponible en [http://dialnet.unirioja.es/servlet/articulo? codigo=3667728], consultado en diciembre de 2011 .

Padró, Carla

2003 "Museología crítica como forma de reflexionar sobre los museos como zonas de conflicto e intercambio", en Jesús-Pedro Lorente Lorente y David Almazán (eds.), Museología crítica y arte contemporáneo, Zaragoza, Prensas Universitarias de Zaragoza, 57-60.

Pardo, Jordi

2000 "Ideas e ideología en el proyecto museológico", Revista Museo (5): 61-71.

Pereyra, Elvira

2006 "Museología, historia, patrimonio y sociedad", en Hildegard K. Vieregg, Mónica Risnicoff de Gorgas, Regina Schiller (eds.), Museología e historia: un campo de conocimiento, sumario analítico-ICOFOM tema 2, XXIX Encuentro Anual del ICOFOM/XV Encuentro Regional del ICOFOM LAM, 5 al 15 de octubre, Córdoba y Alta Gracia, Argentina, documento electrónico disponible en [http://www.museoliniers. org.ar/museologia/conferencias/SumarioAnaliticoTemall-ElviraPereyra.pdf], consultado en diciembre de 2011.

Piaget, Jean 2005 Inteligencia y afectividad, Buenos Aires, Aique.

Puebla, Florencia

2013 Públicos y museos: análisis de visitantes al Museo del Área Fundacional de Mendoza, Mendoza, Facultad de Filosofía y Letras/UNCuyo, CIRSF.
Ruiz Zapatero, Gonzalo

2002 "Arqueología e identidad: la construcción de referentes de prestigio en la sociedad contemporánea", Arqueoweb, Revista sobre Arqueología en Internet, documento electrónico disponible en [http://www.ucm.es/info/arqueoweb/pdf/4-1/ ruizzapatero.pdf], consultado en mayo de 2012.

Sarno, Alicia, María Emilia Grandi y Florencia Lloret 2006 "Museos: ¿nuevos públicos o nuevas actitudes hacia sus públicos?", documento electrónico disponible en [www. geocities.ws/emuseoros/Docs/nuevos_publicos.htm], consultado en noviembre de 2011.

Schmilchuk, Graciela

1997 "El estudio del público. Venturas y desventuras de los estudios de público", Cuicuilco, 3 (7): 31-57.

Shelton, Anthony A.

2011 "De la antropología a la museología crítica y viceversa", Museo y Territorio, 4: 30-40, documento electrónico disponible en [http://www.museoyterritorio.com/pdf/museoyterritorio04-3.pdf], consultado en noviembre de 2011.

Tamanini, Elizabete 1999 "Museu, educação e arqueologia: prospecções entre teoria e prática", Revista do Museu de Arqueologia e Etnologia, 3: 339-345.

Tamiozzo, Azucena 1997 "Historias de una ciudad. La fuente del siglo XIX", El Área Fundacional de Mendoza, 3: 95-155.

Valle Flórez, Rosa

2005 Museos y diversidad: explorando con todos los sentidos, Universidad de León, documento electrónico disponible en [www.portal.unesco.org/culture/en/files], consultado en noviembre de 2011.

Vargas Arenas, Iraida y Mario Sanoja 1993 Historia, identidad y poder, Caracas, Fondo Editorial Tropykos.

Vázquez Olvera, Carlos 2008 "Estudio introductorio. Revisiones y reflexiones en torno a la función social de los museos", Cuicuilco 15 (44): 5-14. 


\section{Resumen}

El presente trabajo trata sobre un estudio de público realizado en el Museo del Área Fundacional de la ciudad de Mendoza (MAF), Argentina, cuyo objeto consistió en indagar cómo los visitantes se relacionaban con el patrimonio histórico-arqueológico expuesto en él. Para ello, se analizaron 2027 visitantes durante los meses de junio-julio del 2011, que aportaron novedosa información que nos llevó a reflexionar, desde la museología crítica, acerca de la labor del museo tanto en el presente como en el futuro.

\section{Palabras clave}

Estudios de público; patrimonio histórico-arqueológico; Museología Crítica

\section{Abstract}

This paper is about a public survey carried out in the Museo de Área Fundacional (MAF, the Foundation Area Museum) in Mendoza, Argentina. The objective was to ascertain how visitors related to the historical and archaeological heritage exhibited in the museum. This was done by analyzing 2027 visitor surveys during June and July 2011, which provided new information leading us to reflect, in critical museology, on the museum's present and future work.

Key words

Visitor surveys; historical and archaeological heritage; Critical Museology

Título en inglés: Public Surveys as a Tool to Analyze the Relation between Society and its Heritage: the Case Study of the Museo [Arqueológico] de Área Fundacional (Foundation Area [Archaeological] Museum) in Mendoza, Argentina 